\title{
An Unusual Cause of Thromboembolism in a 52 Year Old Man
}

\author{
Francesca Zuffada $^{1 *}$, Filippo Airoldi ${ }^{1}$, Andreina Santagostino $^{2}$ and Giuseppe Augello ${ }^{2}$ \\ ${ }^{1}$ Department of Heart, Brain and Vessels, Ospedale di Circolo e Fondazione Macchi, University of Insubria, Italy
}

${ }^{2}$ Electrophysiology and Cardiac Pacing, Istituto Clinico Città Studi, Italy

Received: October 22, 2014; Accepted: March 17, 2015; Published: March 25, 2015

*Corresponding author: Francesca Zuffada, Department of Heart, Brain and Vessels, Ospedale di Circolo e Fondazione Macchi, University of Insubria Viale Borri 57, 21100, Varese, Italy, Tel: +393383362914; E-mail: zuffadafrancesca@gmail.com

\section{Case Report}

A 52-year-old asymptomatic patient was referred to our Centre after performing a pre surgical ECG for nasal septoplasty. The ECG documented isolated negative T wave in aVL and small $r$ wave in the inferior leads (Figure 1). His mother was suffering from hyperhomocysteinemia and he was affected too, so he was treated with folic acid since he was a child. About 20 years before the patient had a previous retinal arterial thrombosis conditioning partial reduction of the visual field. The physical examination was normal. No arrhythmias were discovered during monitoring. A 2D transthoracic echocardiography revealed a slight hypertrophy of a left ventricle with preserved systolic function. Left atrium was not dilated and there was a mobile membrane-like echogenic structure dividing it into two compartments: a proximal one receiving the pulmonary venous flow and a distal one containing left atrial appendage (Figure 2). The microbubbles test, performed through transesophageal echocardiography, showed small inter-atrial right to left shunt (Figure 3). MRI documented the presence of the membrane with course parallel to the mitral valve that separated the atrium into postero-superior and anterior-inferior halves (Figure 4). 3D ultrasound revealed a large opening in the infero-posterior side with a regular flow without hemodynamically significant stenosis (Figure 5). The cine-MRI confirmed the presence of a small leftright jet flow owing to the traction of the inter-atrial septum by the intra-atrial membrane. Due to the huge communication and the absence of symptoms, currently we didn't consider invasive diagnostic tests or surgery intervention for correction of the defects. We decided to put the patient on therapy with low-dose of acetylsalicylic acid and follow him periodically.

\section{Discussion}

Our report emphasizes some observations about Cor Triatriatum Sinister (CTS). This congenital defect is a condition in which the left atrium is divided into two chambers by a fenestrated fibromuscular septum [1].CTSare generally diagnosed in pediatric populations, with age at presentation depending on the size of the membrane fenestration and the resulting obstruction to inflow

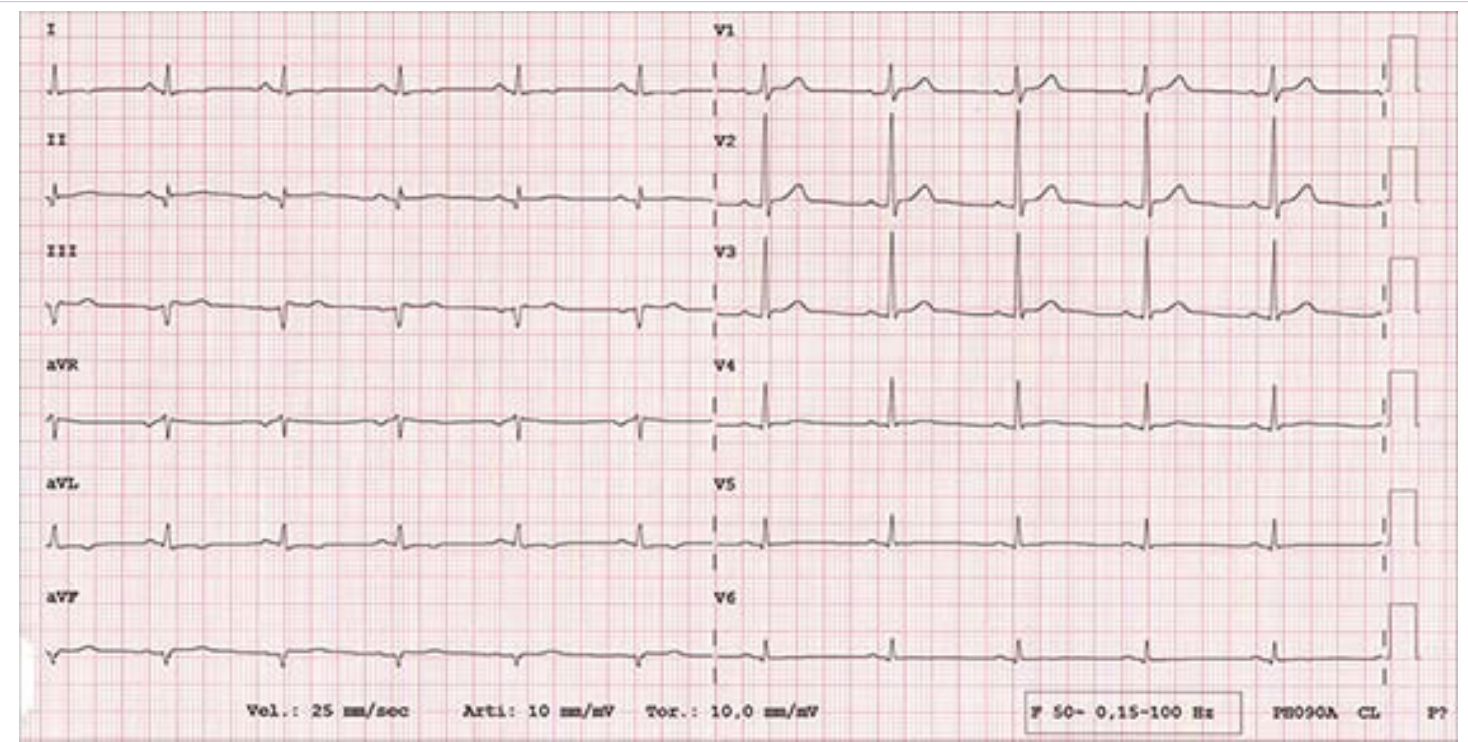

Figure 1: The ECG shows sinus rhythm, normal heart rate and A-V conduction with isolated negative $\mathrm{T}$ wave in aVL and small $\mathrm{r}$ wave in the inferior leads. 


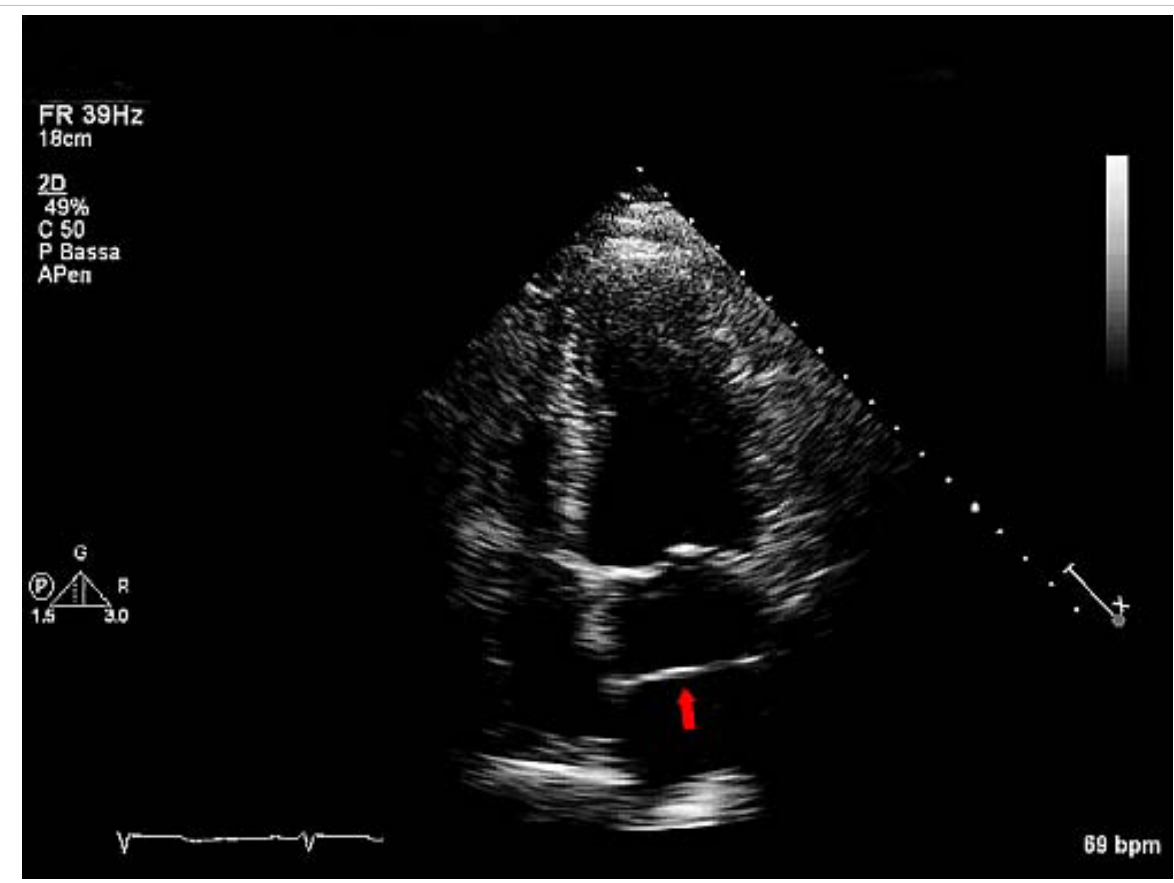

Figure 2: 2D transthoracic echocardiography in apical 4-chamber view: a mobile membrane-like echogenic structure divides the atrium into two parts: a proximal one receiving the pulmonary venous flow and a distal one containing left atrial appendage.

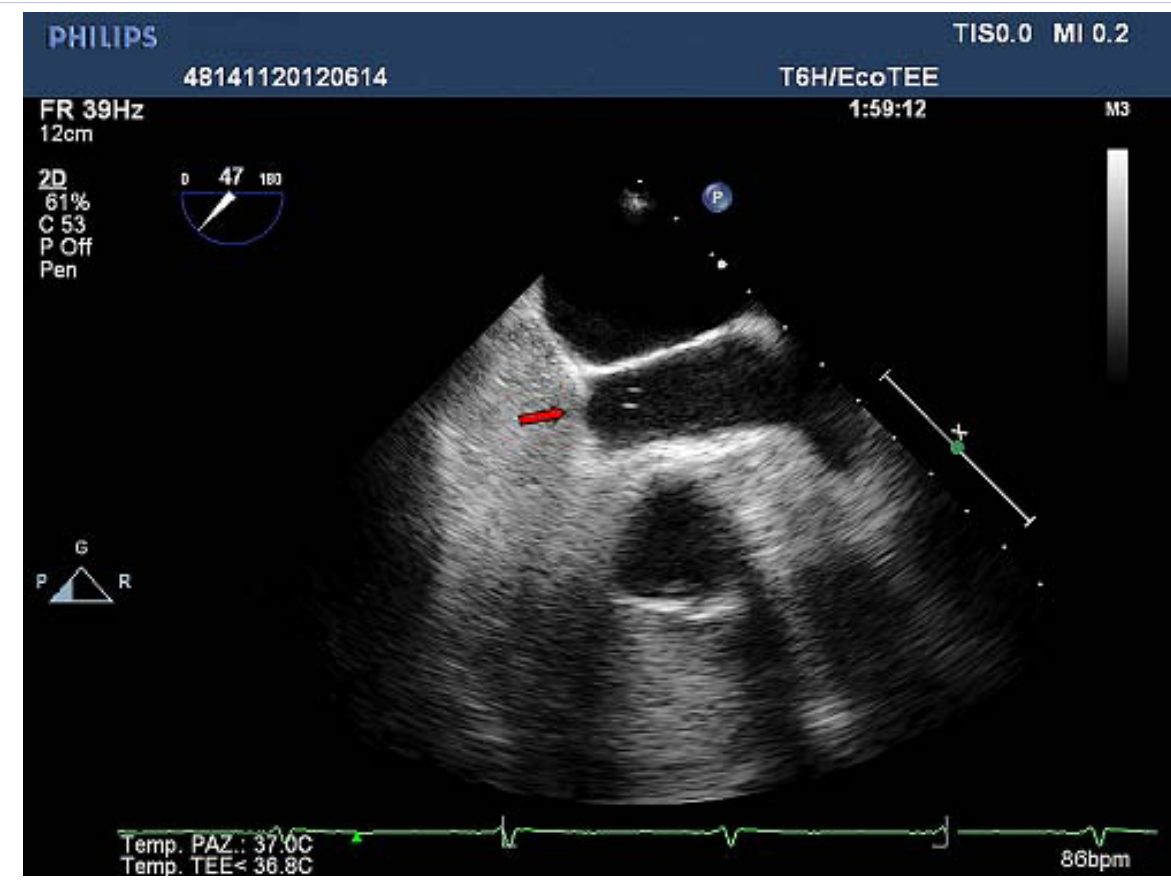

Figure 3: Transesophageal echocardiography $50^{\circ}$ view during microbubbles test, shows small inter-atrial right to left shunt with passage of microbubbles.

[2]. This cardiac anomaly accounts for $0.1 \%$ of cases of congenital heart disease. CTS is often associated with other heart's defects (especially septal defects in $70-80 \%$ of patients) [3,4]. Rarely, the combination of two defects may stay for a long time without symptoms. We think that the previous retinal arterial thrombosis would have been caused by paradoxical embolism through septal defect; this was facilitated by the patient's predisposition to venous thrombosis due to hyperhomocysteinemia history. Unfortunately the echocardiogram wasn't been made at the time of retinal thrombosis. CTS diagnosis is usually established by 2D transthoracic echocardiography. Transesophageal echocardiography provides better imaging of the left atria, left 


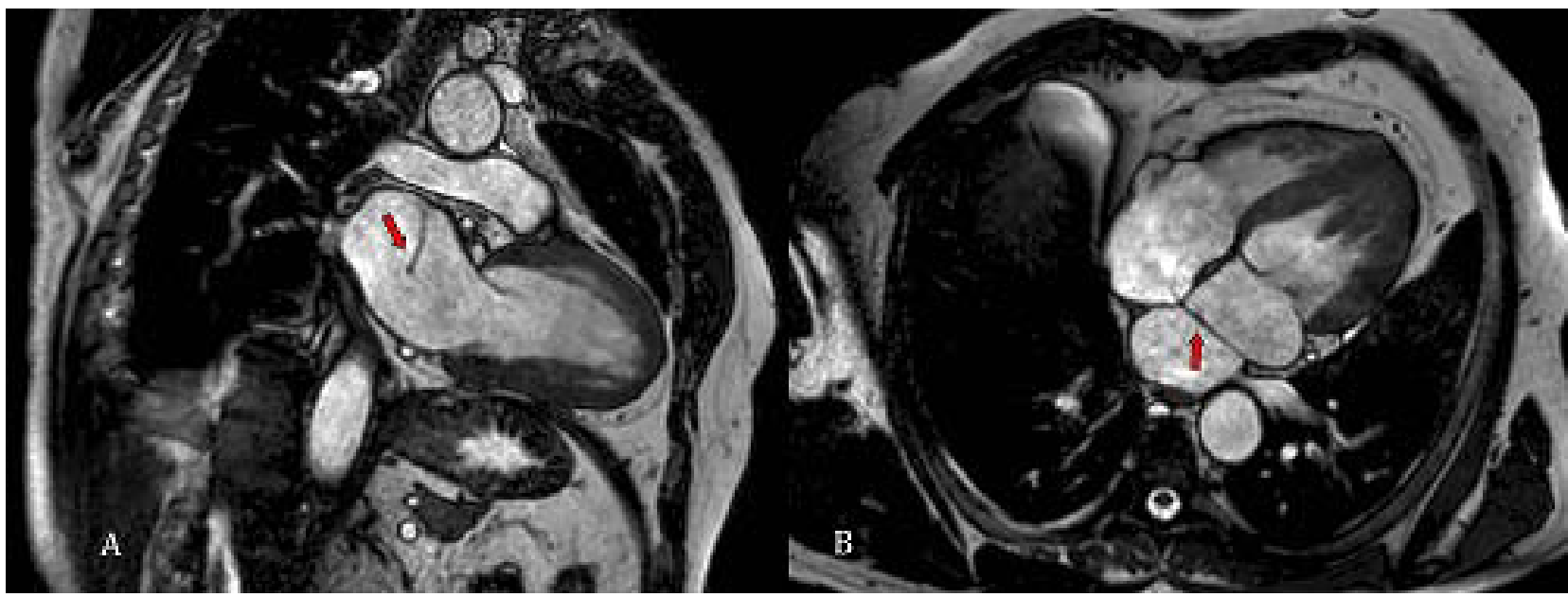

Figure 4: Heart MRI in 2 and 4-chamber view. The atrial membrane is parallel to the mitral valve and divides the atrium into posterosuperior and anterior-inferior halves; it's also possible to see in 2-chamber view the large membrane discontinuity which allows communication.

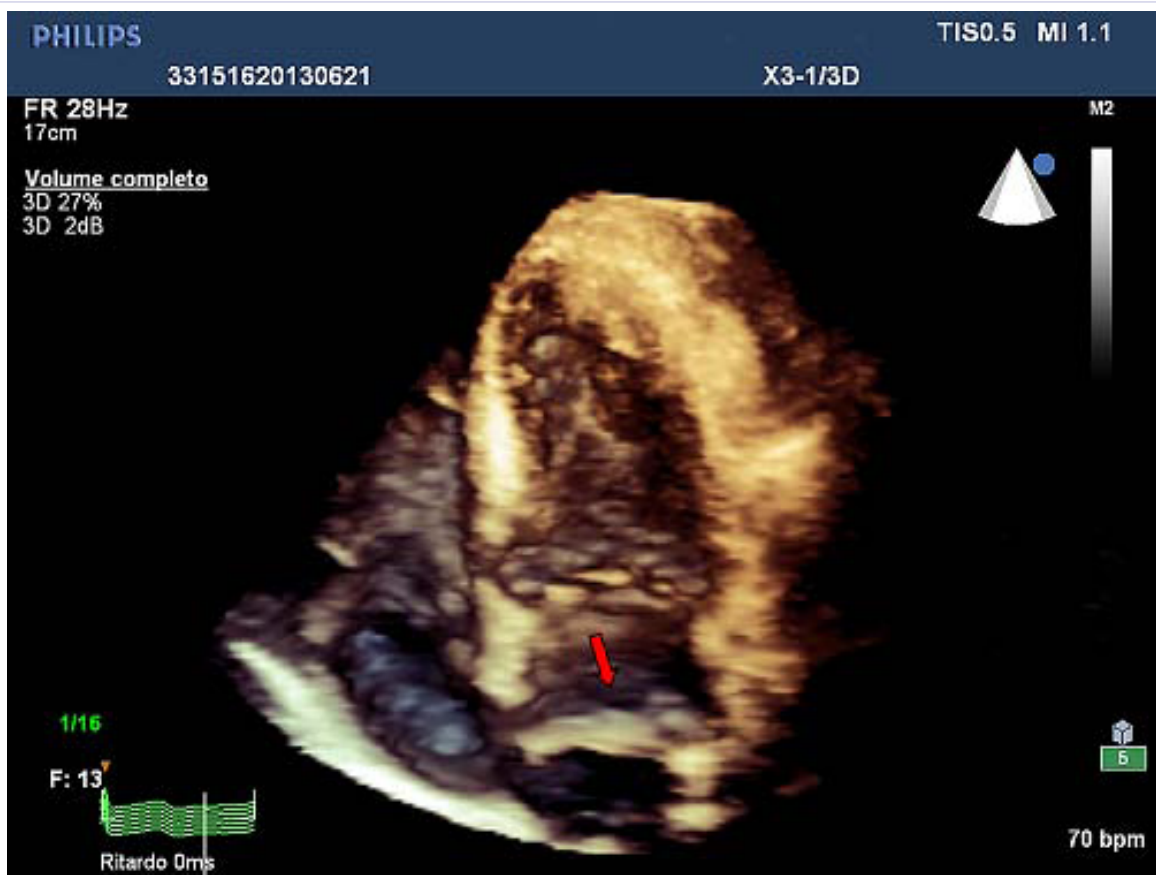

Figure 5: Anatomic reconstruction in 4-chamber view, using 3-D transthoracic echocardiography, reveals a large opening in the inferoposterior side of the membrane.

atrial appendage, morphology of the dividing membrane and the degree of obstruction [2]. 3D echocardiography is a more recent diagnostic tool providing additional information, able to demonstrate the entire membrane, the size, the location and the number of openings in the dividing membrane $[2,5,6]$. Finally, the MRI allows getting more and more detailed images, especially in pre-surgical setting. Cine-MRI clearly depicts the fenestration within the membrane with the associated flow turbulence, seen as a low intensity signal contrasted with the high-signal intensity of normal blood flow $[5,7,8]$. In conclusion, we hope that in all cases of peripheral embolism a transthoracic echocardiogram is performed as first line imaging approach to rule out favoring cardiac abnormalities.

\section{References}

1. Richardson J V, Doty DB, Siewers R, Zuberbuhler JR. Cor triatriatum (subdivided left atrium). J Thorac Cardiovasc Surg. 1981; 81 (2): 232-8.

2. Malik A, Fram D, Mohani A, Fischerkeller M, Yekta A, Mohyuddin Y, et al. Cor triatriatum: a multimodality imaging approach. Can J Cardiol. 2008, 24(3): e19-20.

3. Hamdan R, Mirochnik N, Celermajer D, Nassar P, Iserin L. Cor 
Triatriatum Sinister diagnosed in adult life with three dimensional transesophageal echocardiography. BMC Cardiovascular Disord. 2010; 10: 54. doi: 10.1186/1471-2261-10-54.

4. Krasermann Z, Scheld HH, Tjan TD, Krasermann T. Cor triatriatum: short review of the literature upon ten new cases. Hertz. 2007; 32(6): 506-10.

5. Penafiel HP, Yeo TC. Cor triatriatum presenting in adulthood: a case report. Int J Cardiol. 2008; 123(2): e25-7.

6. Thakrar A, Shapiro MD, Jassal DS, Neilan TG, King ME, Abbara S. Cor triatriatum: the utility of cardiovascular imaging. Can J Cardiol. 2007; 23(2): 143-5.

7. Kadner A, Meszaros K, Mueller C, Schoenhoff F, Hutter D, Carrel T. Cor triatriatum sinister. Multimed Man Cardiothorac Surg. 2014; doi: 10.1093/mmcts/mmu005. Print 2014.

8. Bezgin T, Canga Y, Karagöz A, Yılmaz F, Doğan C, Elveran A, et al. Multimodality imaging of cor triatriatum sinister in an octagenerian. Echocardiography. 2014; 31(8): E254-6. doi: 10.1111/echo.12660. 